Internet Engineering Task Force (IETF)

F. Wei

Request for Comments: 6232

Updates: 5301, 5304, 5310

Category: Standards Track

Y. Qin

ISSN: $2070-1721$

Z. Li

China Mobile

T. Li

Cisco Systems, Inc.

J. Dong

Huawei Technologies

May 2011

Purge Originator Identification TLV for IS-IS

Abstract

At present, an IS-IS purge does not contain any information

identifying the Intermediate system (IS) that generates the purge.

This makes it difficult to locate the source IS.

To address this issue, this document defines a TLV to be added to purges to record the system ID of the IS generating it. Since normal Link State Protocol Data Unit (LSP) flooding does not change LSP contents, this TLV should propagate with the purge.

This document updates RFC 5301, RFC 5304, and RFC 5310.

Status of This Memo

This is an Internet Standards Track document.

This document is a product of the Internet Engineering Task Force (IETF). It represents the consensus of the IETF community. It has received public review and has been approved for publication by the Internet Engineering Steering Group (IESG). Further information on Internet standards is available in section 2 of RFC 5741.

Information about the current status of this document, any errata, and how to provide feedback on it may be obtained at http://www.rfc-editor.org/info/rfc6232. 
Copyright Notice

Copyright (c) 2011 IETF Trust and the persons identified as the document authors. All rights reserved.

This document is subject to BCP 78 and the IETF Trust's Legal Provisions Relating to IETF Documents

(http://trustee.ietf.org/license-info) in effect on the date of publication of this document. Please review these documents carefully, as they describe your rights and restrictions with respect to this document. Code Components extracted from this document must include Simplified BSD License text as described in section $4 . e$ of the Trust Legal Provisions and are provided without warranty as described in the Simplified BSD License.

Table of Contents

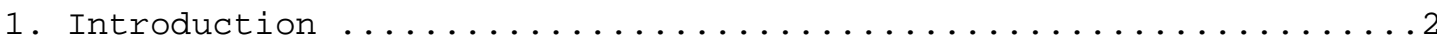

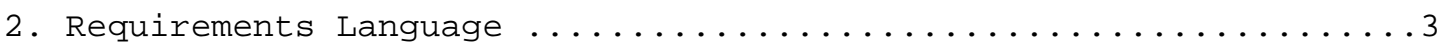

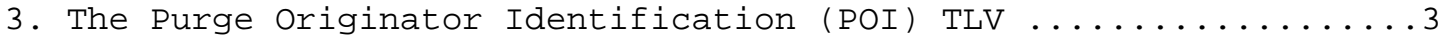

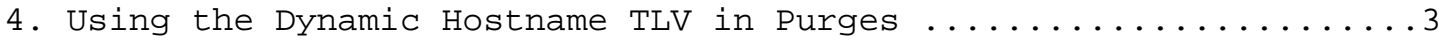

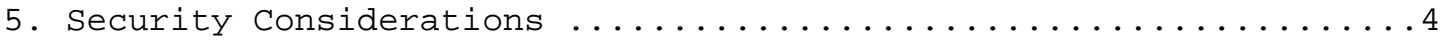

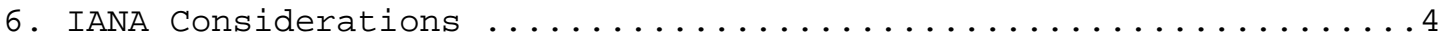

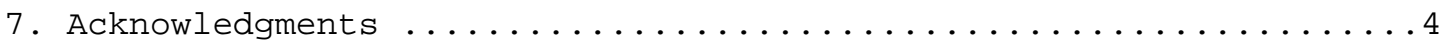

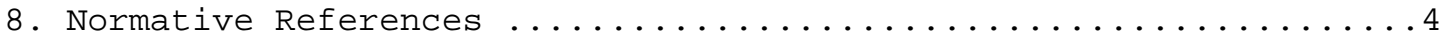

1. Introduction

The IS-IS [ISO-10589] routing protocol has been widely used in largescale IP networks because of its strong scalability and fast convergence.

The IS-IS protocol floods purges throughout an area, regardless of which IS initiated the purge. If a network operator would like to investigate the cause of the purge, it is difficult to determine the origin of the purge. At present, the IS-IS protocol has no mechanism to locate the originator of a purge. To address this problem, this document defines a TLV to be added to purges to record the system ID of the IS generating the purge.

Field experience has shown several circumstances where an IS can improperly generate a purge. These are all due to implementation deficiencies or implementations that predate [ISO-TC1] and generate a purge when they receive a corrupted Link state Protocol Data Unit $(\mathrm{LSP})$. 


\section{Requirements Language}

The key words "MUST", "MUST NOT", "REQUIRED", "SHALL", "SHALL NOT", "SHOULD", "SHOULD NOT", "RECOMMENDED", "MAY", and "OPTIONAL" in this document are to be interpreted as described in [RFC2119].

3. The Purge Originator Identification (POI) TLV

This document defines a TLV to be included in purges. If an IS generates a purge, it SHOULD include this TLV in the purge with its own system ID. If an IS receives a purge that does not include this TLV, then it SHOULD add this TLV with both its own system ID and the system ID of the IS from which it received the purge. This allows ISs receiving purges to log the system ID of the originator, or the upstream source of the purge. This makes it much easier for the network administrator to locate the origin of the purge and thus the cause of the purge. Similarly, this TLV is helpful to developers in lab situations.

The POI TLV is defined as:

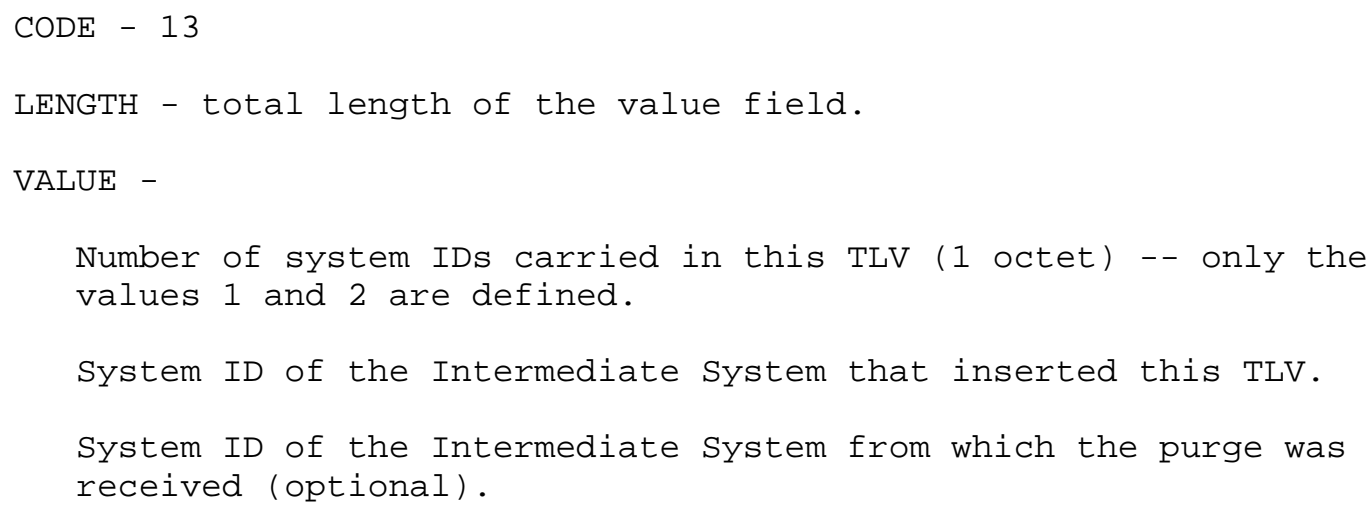

The POI TLV SHOULD be found in all purges and MUST NOT be found in LSPs with a non-zero Remaining Lifetime.

4. Using the Dynamic Hostname TLV in Purges

This document also extends the use of the Dynamic hostname TLV (type 137) [RFC5301] to further aid in the rapid identification of the system that generated the purge. This TLV MAY be included in purges. Implementations SHOULD include one instance of the Dynamic hostname TLV if the POI TLV is included. Only the local hostname should be inserted. 


\section{Security Considerations}

Use of the extensions defined here, with authentication as defined in [RFC5304] or [RFC5310], will result in the discarding of purges by legacy systems that are in strict conformance with either of those RFCs. This may compromise the correctness/consistency of the routing database unless all ISs in the network support these extensions. Therefore, all implementations in a domain implementing authentication MUST be upgraded to receive the POI TLV before any IS is allowed to generate a purge with the POI TLV.

More interactions between the POI TLV, the Dynamic hostname TLV, and the Authentication TLV are described in [RFC6233].

6. IANA Considerations

IANA has assigned code point 13 for the 'Purge Originator Identification' TLV from the IS-IS 'TLV Codepoints' registry. The additional values for this TLV should be IIH:n, LSP:y, SNP:n, and Purge:y.

\section{Acknowledgments}

Many thanks to Adrian Farrel and Daniel King for their comments to improve this document and move it forward.

The first version of this document was mainly composed by Lianyuan Li.

Acknowledgments are given to the discussion in the mailing list. Some improvements to this document are based on the discussion.

8. Normative References

[ISO-10589] ISO, "Intermediate system to Intermediate system intra-domain routeing information exchange protocol for use in conjunction with the protocol for providing the connectionless-mode Network Service (ISO 8473)", ISO/IEC 10589:2002.

[ISO-TC1] ISO, "Intermediate system to Intermediate system intra-domain routeing information exchange protocol for use in conjunction with the protocol for providing the connectionless-mode Network Service (ISO 8473) -Technical Corrigendum 1", ISO/IEC 10589:1992/ Cor. $1: 1993$. 
[RFC2119] Bradner, S., "Key words for use in RFCs to Indicate Requirement Levels", BCP 14, RFC 2119, March 1997.

[RFC5301] McPherson, D. and N. Shen, "Dynamic Hostname Exchange Mechanism for IS-IS", RFC 5301, October 2008.

[RFC5304] Li, T. and R. Atkinson, "IS-IS Cryptographic Authentication", RFC 5304, October 2008.

[RFC5310] Bhatia, M., Manral, V., Li, T., Atkinson, R., White, R., and M. Fanto, "IS-IS Generic Cryptographic Authentication", RFC 5310, February 2009.

[RFC6233] Li, T. and L. Ginsberg, "IS-IS Registry Extension for Purges", RFC 6233, May 2011. 
Authors' Addresses

Fang Wei

China Mobile

No. 29, Financial Street, Xicheng District

Beijing 100032

P.R. China

EMail: weifang@chinamobile.com

Yue Qin

China Mobile

No. 29, Financial Street, Xicheng District

Beijing 100032

P.R. China

EMail: qinyue@chinamobile.com

Zhenqiang Li

China Mobile

Unit2, Dacheng Plaza, No. 28 Xuanwumenxi Ave., Xuanwu District Beijing 100053

P.R. China

EMail: lizhenqiang@chinamobile.com

Tony Li

Cisco Systems, Inc.

170 W. Tasman Dr.

San Jose, CA 95134

USA

EMail: tony.li@tony.li

Jie Dong

Huawei Technologies

Kuike Building, No. 9 Xinxi Rd., Haidian District

Beijing 100085

P.R. China

EMail: dongjie_dj@huawei.com 